\title{
Observation of a Single-Beam Gradient Force Acoustical Trap for Elastic Particles: Acoustical Tweezers
}

\author{
Diego Baresch, ${ }^{1}$ Jean-Louis Thomas, ${ }^{2, *}$ and Régis Marchiano ${ }^{3}$ \\ ${ }^{1}$ Sorbonne Universités, UPMC Univ Paris 06, CNRS, UMR 7588, Institut des NanoSciences de Paris, F-75005 Paris, France \\ ${ }^{2}$ CNRS, UMR 7588, Institut des NanoSciences de Paris, F-75005 Paris, France \\ ${ }^{3}$ Sorbonne Universités, UPMC Univ Paris 06, CNRS, UMR 7190, Institut Jean le Rond d'Alembert, F-75005 Paris, France
}

(Received 25 June 2015; published 11 January 2016)

\begin{abstract}
We demonstrate the trapping of elastic particles by the large gradient force of a single acoustical beam in three dimensions. Acoustical tweezers can push, pull and accurately control both the position and the forces exerted on a unique particle. Forces in excess of 1 micronewton were exerted on polystyrene beads in the submillimeter range. A beam intensity less than $50 \mathrm{~W} / \mathrm{cm}^{2}$ was required, ensuring damage-free trapping conditions. The large spectrum of frequencies covered by coherent ultrasonic sources provides a wide variety of manipulation possibilities from macroscopic to microscopic length scales. Our observations could open the way to important applications, in particular, in biology and biophysics at the cellular scale and for the design of acoustical machines in microfluidic environments.
\end{abstract}

DOI: 10.1103/PhysRevLett.116.024301

Ashkin observed that the radiation pressure of a focused laser could significantly accelerate particles in the direction of light propagation [1] — an axial acceleration that results from momentum conservation and light reflection by the particle. Importantly, an additional unanticipated force transversally attracted the particles towards the beam axis where they were successively optically guided. This component of radiation pressure was called gradient force. Axial pushing forces could be canceled by the wall of the chamber or by a second counterpropagating beam in order to obtain a stable equilibrium position. Only two decades later did the first single-beam gradient trap appear, a specific scheme dubbed optical tweezers (OTs) [2,3]. OTs can attract and stably trap a particle at a single equilibrium position in space, the beam focus. Defying intuition, the light beam exerts a negative pulling force on a particle located downstream from the focus. This restoring force is the axial counterpart of the lateral gradient force initially observed. The particles can be displaced with nanometer accuracy by simply moving the light beam mechanically. These unique features of OTs lead to dexterous contactless manipulation in three dimensions and have had a wide and far-reaching impact since their inception, spanning the range from life science, material science and microfluidics to laser cooling of atoms [4-8]. In particular, precisely calibrated OTs have enabled force measurements with piconewton accuracy on single molecules and opened up important new areas in biophysics [9].

An early recognized issue is the heating of both the object and the trapping medium [10]. Radiation pressure is proportional to the wave field intensity divided by its speed of propagation, $I / c$. Hence, light intensities of about $10^{7}-10^{8} \mathrm{~W} / \mathrm{cm}^{2}$ at the focus yield weak forces in the $\mathrm{pN}$ range on micron-sized objects [11]. From this point of view, introducing ultrasonic traps is relevant. The speed of sound thus gives a major advantage to acoustics ( 5 orders of magnitude on the force), and the change in nature of the oscillating field opens up the prospect of operating in media generally exposed to heating by light. Furthermore, piezoelectric sources with high efficiency are available with frequencies from $\mathrm{kHz}$ up to $\mathrm{GHz}$, scaling macroscopic to microscopic wavelengths, broadening the range of particle sizes that can be trapped as well as the operating distances from the source.

Acoustic levitation traps were developed independently and concomitantly with their optical counterpart [12-14]. Recent improvements to standing acoustic wave schemes led to levitating and translating single or multiple particles in air [15], and acoustophoresis provides advanced particle, cell and organism separation in complex microfluidic environments [16,17]. Standing wave schemes have recently been proposed to accurately manipulate particles in two dimensions using surface $[18,19]$ or bulk acoustic waves [20] with phase or frequency shifts in order to demonstrate capabilities similar to OTs. However, all the aforementioned techniques share the same limitations; e.g., standing waves form multiple equilibrium positions in one or two dimensions, each of which is likely to trap one or various particles at the same time, therefore precluding separability and selectivity at the single particle level with ease [21,22].

Obtaining a three-dimensional restoring force by tightly focusing an acoustic beam has proven to be challenging: $\mathrm{Wu}$ reported on a dual beam trap [23], whereas lateral manipulation with an ultrasonic beam was successfully implemented while the axial expelling force was canceled by a membrane $[24,25]$. Both concepts are similar to those reported in Ashkin's seminal work on particle acceleration [1]. 
Here we report on the first observation of a negative gradient pulling force with acoustic waves and hence the achievement of all acoustical single beam trapping, i.e., acoustical tweezers.

Acoustic radiation force.-From a previous analysis [26], we inferred that failure to trap in previous attempts was expected with axisymmetric beams. Indeed, solid elastic particles have a propensity to be expelled from intensity maxima at the focal spot of a conventional focused ultrasonic beam. Starting from recent developments of the theory for acoustic radiation force (ARF) calculations [27,28] and the acoustics analogous to the generalized Lorenz-Mie theory (GLMT) [29,30], it can be shown (see Ref. [31]) that for small spherical particles (radius $a$ ) compared to the wavelength $(\lambda)$, the ARF takes the following form:

$$
\begin{aligned}
\vec{F}= & -\frac{1}{2}\left\{\operatorname{Re}\left(\alpha_{m}\right) \frac{1}{2 \rho c^{2}} \vec{\nabla}|p|^{2}-\operatorname{Re}\left(\alpha_{d}\right) \frac{1}{2} \rho \vec{\nabla}|\vec{v}|^{2}\right. \\
& +\left(\frac{k}{c} \operatorname{Im}\left(\alpha_{m}\right)-\frac{k^{4}}{12 \pi c} \operatorname{Re}\left(\alpha_{m}\right) \operatorname{Re}\left(\alpha_{d}\right)\right) \operatorname{Re}\left(p \vec{v}^{*}\right) \\
& \left.+\rho \operatorname{Im}\left(\alpha_{d}\right) \operatorname{Im}\left((\vec{v} \cdot \vec{\nabla}) \vec{v}^{*}\right)\right\},
\end{aligned}
$$

where $p$ and $\vec{v}$ are the first-order acoustic pressure and particular velocity fields, respectively; $\rho$ is the host fluid's density and $k=2 \pi f / c$ ( $f$ is the frequency and $c$ is the wave speed). Re and Im denote the real and imaginary parts of these complex fields while ${ }^{*}$ stands for complex conjugation. $\alpha_{m}=$ $\alpha_{m}^{0} /\left[1+i\left(k^{3} / 4 \pi\right) \alpha_{m}^{0}\right]$ and $\alpha_{d}=\alpha_{d}^{0} /\left[1-i\left(k^{3} / 12 \pi\right) \alpha_{d}^{0}\right]$ are two acoustic strength parameters associated with the sphere's monopolar and dipolar modes, where

$$
\begin{aligned}
\alpha_{m}^{0} & =\frac{4}{3} \pi a^{3}\left(1-\frac{\rho c^{2}}{\rho_{p}\left(c_{l}^{2}-\frac{4}{3} c_{t}^{2}\right)}\right), \\
\alpha_{d}^{0} & =4 \pi a^{3}\left(\frac{\rho_{p}-\rho}{2 \rho_{p}+\rho}\right) .
\end{aligned}
$$

Here, $c_{l}$ and $c_{t}$ are the longitudinal and transverse propagation speeds in the particle of density $\rho_{p}$. The first two terms in Eq. (1) stand for an acoustic gradient force that is tantamount to its optical counterpart, while the remaining terms form the scattering force. For most of the elastic particles suspended in liquids, the gradient term associated with an axisymmetric beam points towards energy density minima precluding from establishing a trapping behavior. However, we have recently proposed that shaping the beam's wave front may restore three-dimensional trapping forces [26] as a consequence of a monopolar mode annihilation on the propagation axis (see Ref. [31]). Focused acoustical vortices satisfy various constraints to retrieve both lateral and axial restoring gradient forces.

Field synthesis.-These peculiar wave fields have been successfully generated in acoustics [39-41] and applied to investigate the transfer of orbital angular momentum to matter [42-46]. In Fig. 1(a), a sketch of the experimental setup is shown. A multielement array of piezoelectric transducers is driven by independent arbitrary signal generators to synthesize the beam in a large water tank. A tight focusing of the vortex is obtained with a high focal ratio acoustical lens. Based on numerical calculations [26], an optimal acoustical vortex is determined and used as an input to a versatile and robust wave-front synthesis technique [40]. The array of transducers is driven by the calculated signals to experimentally generate the incident field. We drive the transducers at a frequency $f=$ $1.15 \mathrm{MHz}$ corresponding to a wavelength $\lambda=1.3 \mathrm{~mm}$ in water. A hydrophone scanning a grid of points defined near the focus measures the instantaneous pressure field. The field's normalized intensity in the direction of propagation is shown in Fig. 1(b). The depth of field is approximatively equal to $4 \lambda=5.2 \mathrm{~mm}$ and will determine the axial trapping extent of the tweezers. In the focal plane [Fig. 1(c)-1(e)], the acoustical intensity is focused to a "ring" pattern that is a peculiarity of vortex beams. Looking at the phase diagram [Fig. 1(c)], its variation from $-\pi$ to $\pi$ around the beam axis is characteristic of a vortex of topological charge equal to 1 [39-41]. The wave front describes a single helix with a pitch equal to $\lambda$ and is left handed. Measured and predicted pressure fields are in perfect agreement [see Fig. 1(e) and Figs. S1 and S2 in Ref. [31]). When the emitter is driven at maximum power, the peak value of the pressure on the ring reaches $0.8 \pm 0.1 \mathrm{MPa}$ and the acoustical intensity of approximatively $42 \mathrm{~W} / \mathrm{cm}^{2}$ remains weak at the focus.

Acoustical tweezers.-In this vertical configuration [Fig. 1(a)], the ultrasonic emitter focuses the beam upon a thin polyethylene film (largely transparent to acoustic waves) on which the polystyrene particles are dispersed (their diameters range from $190 \mu \mathrm{m}$ to $390 \mu \mathrm{m}$ ). The film, mounted on a three-axis positioning system, allows us to move the particles around the region of interest. As the downward propagating beam impinges a particle, the latter is lifted by a strong negative gradient force and remains stably trapped in three dimensions. A photograph of a levitated particle (radius $a=170 \mu \mathrm{m}$ ) is shown in Fig. 2(a) (see Video 1 in Ref. [31] as well). The predicted behavior of the axial force is shown in Fig. 2(b). It is negative beneath the beam focus (lifting force) and positive above (pushing force). Nevertheless, the particle reaches an axial equilibrium position when the negative radiation force balances adverse effects acting in the direction of propagation, and the axial equilibrium position is generally below the beam's focus (typically $30.0 \mathrm{~mm}$ away from the lens). These opposite forces are the positive axial scattering force, the gravity and the viscous drag force resulting from acoustic streaming, that is to say, a flow generated by absorption of the beam's intensity in the bulk of the fluid $[47,48]$. The only weight of the sphere represents $20 \mathrm{nN}$ for the largest polystyrene particles. The axial range of operation of the tweezers is investigated by changing the distance 
(a)

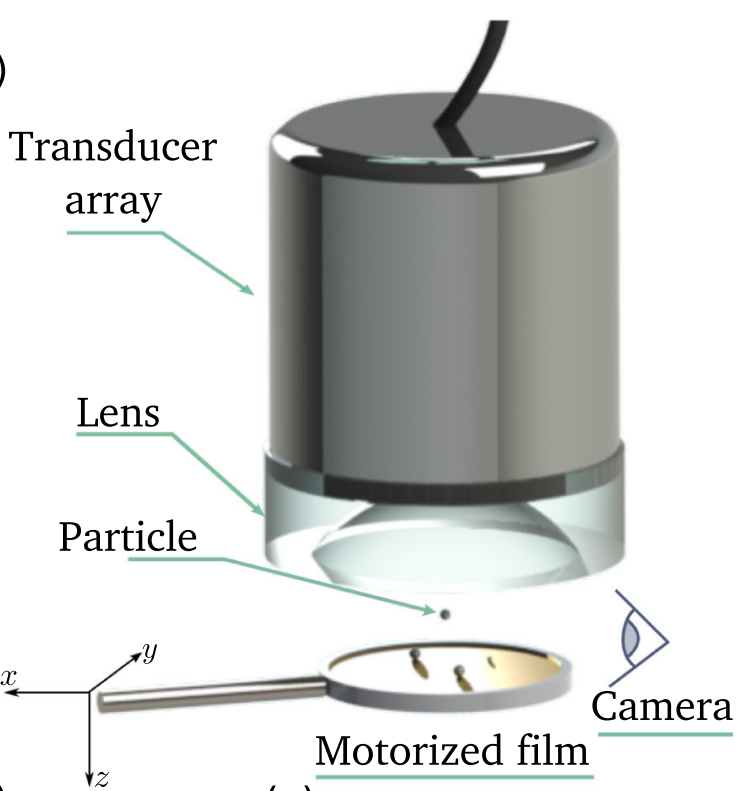

(b)

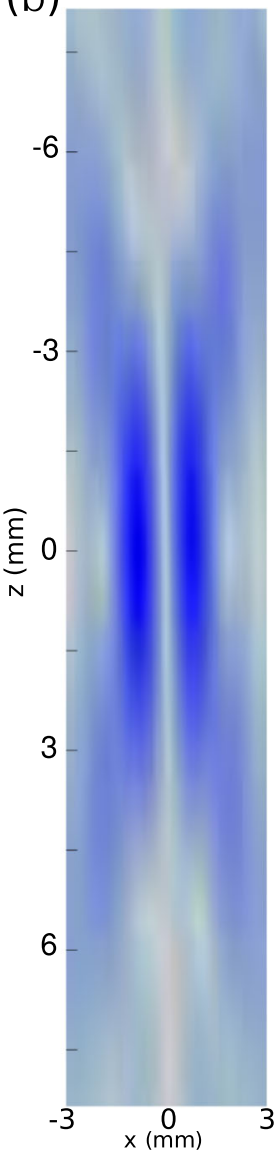

(c)

(d)
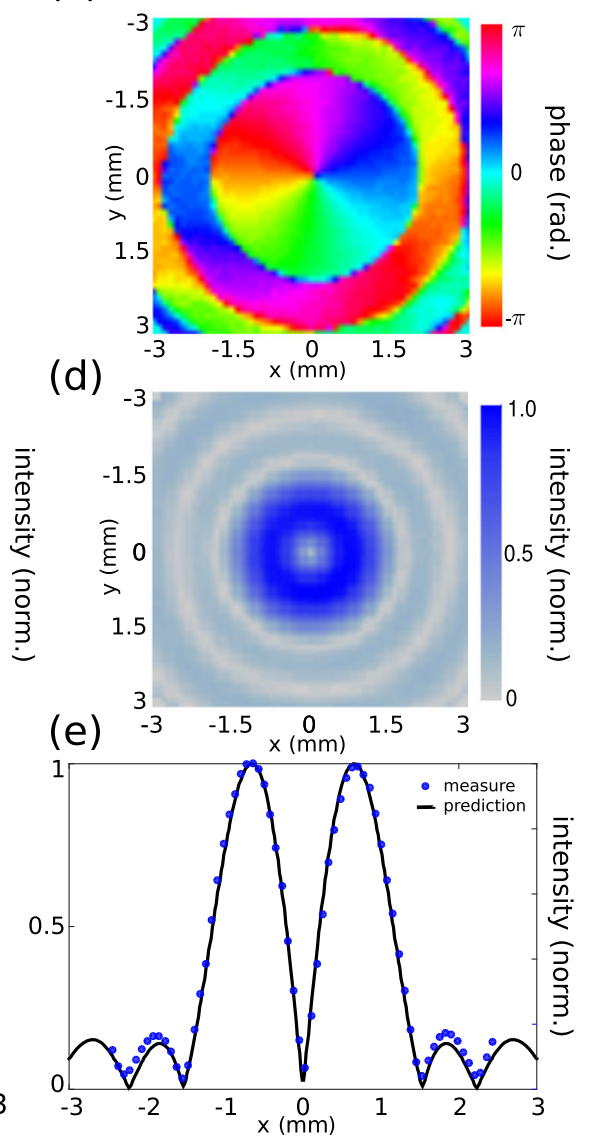

FIG. 1. (a) Sketch of the ultrasonic emitter (1.15 MHz) immersed in a water tank. The beam is focused with a concave acoustical lens of focal ratio $\simeq 0.7$ upon polystyrene particles lying on an acoustically transparent polyethylene film, (b)-(e) Measured acoustic field. (b) Color plot of the normalized acoustic intensity in the direction of propagation (negative to positive $z$ ). (c,d) Field's phase and intensity in the focal plane $(z=0)$. (e) Comparison of the measured and predicted pressure fields (intensity) in the focal plane $z=0 \mathrm{~mm}$.

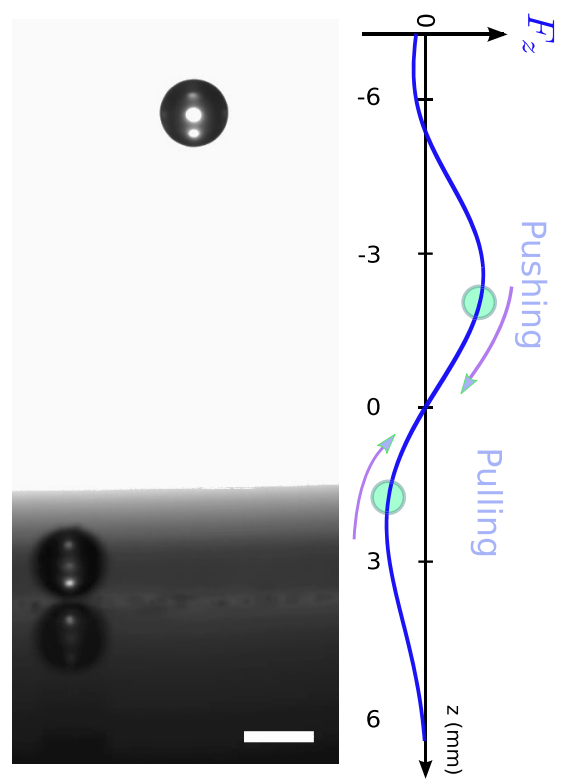

(a)

(b)

FIG. 2. (a) Photograph of a particle of radius $a=170 \mu \mathrm{m}$ trapped in three dimensions. The large negative axial radiation force lifts the particle from a distance $\simeq 2.6 \mathrm{~mm}$ below the equilibrium position. A second particle lying on the film is not attracted to the focus. The scale bar represents $200 \mu \mathrm{m}$. (b) Theoretical prediction of the axial acoustical radiation force as a function of the particle's position on the propagation axis.

separating the particle and the beam focus before switching the source on. The maximum lifting distance was found to reach $3.4 \mathrm{~mm}$ or equivalently $\simeq 2.6 \lambda$ beneath the focus for the maximum emission intensity $\left(I=42 \mathrm{~W} / \mathrm{cm}^{2}\right)$. Contrarily, if the particle is initially located above the equilibrium position, it is pressed against the film by a positive axial force.

The lateral component of the radiation force is predicted to be at least 1 order of magnitude stronger than the axial counterpart [see Fig. S3 in Ref. [31]]. So, when the particle is initially located aside the beam axis, a large lateral acceleration is observed before it starts to lift. Acquiring images at a high frame rate (520 fps), we recorded the motion of the particle as it was attracted towards the central beam core. By taking into account the viscous drag force and other inertial forces arising from the particle's acceleration, we calculated the net lateral force acting on the particle (see Fig. 3 and Ref. [31]). For a polystyrene particle of radius $a=190 \mu \mathrm{m}$, the lateral acoustic radiation force reaches $F_{\rho} \simeq 1.5 \mu \mathrm{N}$ at a lateral distance $\rho=370 \mu \mathrm{m}$ away from the propagation axis. Measurements and force predictions [29] are in good agreement. Nevertheless, various improvements could be reached by precisely accounting for the effect of the film on the bead's lateral acceleration and measuring the absolute pressure field with better accuracy. As in this region the radiation force varies linearly with the lateral displacement, this corresponds to 


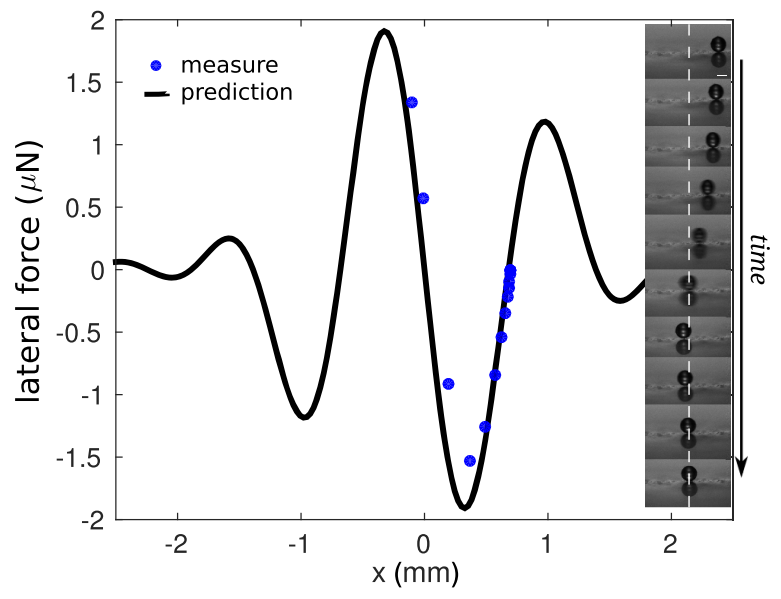

FIG. 3. Measurement of the lateral force as a particle is accelerated towards the propagation axis. The force is obtained as a function of the lateral position $x$ by calculating the lateral acceleration from the instantaneous position of the center of the sphere. The snapshots are displayed every $2 \mathrm{~ms}$.

an approximate lateral trap stiffness of $\kappa \simeq 4 \mathrm{nN} / \mu \mathrm{m}$. The maximum lateral displacement reached $\rho_{\max }=700 \mu \mathrm{m}$ for various particles. If the particle is initially located beyond, the positive lateral force will expel it instead. This remarkable selectivity feature ensures an accurate operation at the single particle level in media with high densities of particles (see Video 2 in Ref. [31]). If a unique particle is trapped at the focus, a potential barrier will keep the others away from the equilibrium position. Note that this is not the case for most optical trapping experiments where many particles can quickly collect at the focus for a high concentration of particles $[3,10]$. Nevertheless, if two particles are located close enough to each other, the beam simultaneously lifts both (see Video 3 in Ref. [31]). Systems of two trapped particles are shown in Fig. 4. It can be seen that both particles will react to the primary incident beam and be trapped in a single equilibrium position but can additionally exhibit a mutual interplay. Depending on the relative size of each particle, they can either attract or repulse each other and maintain a separation distance $d \simeq$ $0.25 \lambda$ [Fig. 4(c)]. While a systematic study of multiple particle interactions is beyond the scope of this study, such systems may offer interesting possibilities to probe the accuracy of recent radiation force calculations for multiple scatterers [49].

To demonstrate transportation, once the particle is picked up, the film is very rapidly moved to another position where the particle is precisely and gently dropped in a cavity (see Video 4 in Ref. [31]). The streams generated by the highspeed translation of the film $(50 \mathrm{~mm} / \mathrm{s})$ do not affect the position of the trapped particle. Reversely, this observation implies the ability to rapidly move the trapped specimen in the host medium, displacing the trapping beam instead, and suggests that the tweezers are relevant for high-speed manipulation tasks.
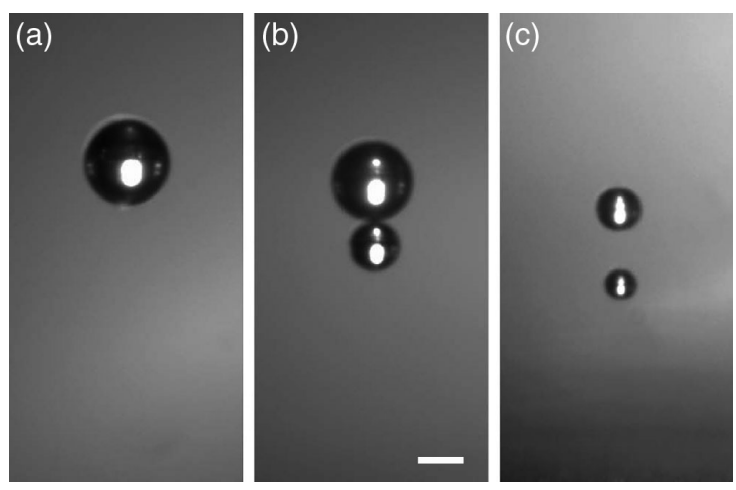

FIG. 4. Systems of one or two particles can be trapped. (a) Radius $a=189 \mu \mathrm{m}$. (b) Radii $a=177$ and $108 \mu \mathrm{m}$. (c) Radii $a=98$ and $67 \mu \mathrm{m}$. The scale bar represents $200 \mu \mathrm{m}$. Their selfarrangement exhibits the effect of secondary acoustic radiation forces.

Another interesting possibility is to precisely control the dynamics of the particle to fashion an actuator for various applications. A first and simple way to manage this is to control successive cycles of catching and dropping of the particle by switching the power source on and off at different rates (see Video 5 in Ref. [31]). The incident power is driven with a square signal of three different periods: $0.3,0.15$, and $0.1 \mathrm{~s}$. Thus, we can control the amplitude and the frequency of the oscillations of a particle around its equilibrium position. This could be used, for instance, to initiate streams in the host fluid. While not exploited here, the versatility of the wave-front synthesis technique offers many possibilities to reconfigure in real time the form and the position of the trapping beam as in holographic optical traps [5,50,51]. Note that, in all experiments, once the particle is trapped, we completely remove the film to make sure it does not disturb the trapping force.

Discussion.-For the first reported time, the results demonstrate remote trapping of elastic particles in three dimensions with the gradient force of a single acoustic beam, i.e., acoustical tweezers. We exerted forces overtaking those of optical tweezers by 3 to 4 orders of magnitude while reducing the intensity flux by 5 . Acoustical tweezers can selectively pick and control the position of a particle in all three dimensions. A mechanical positioning of the beam focus with a translational stage will ensure a precise control of the particle's position. If the source is made of a set of independent transducers, electronic steering of the beam is feasible, such as spatial light modulators in optics [50]. Moreover, acoustical vortices carry a quantified orbital angular momentum $[39,40]$ that can be transferred to matter [42-46,52,53], so controlled rotation of absorbing particles is expected to provide a 4th degree of freedom for contactless manipulation and forms the basis of an undergoing study.

Concerning different materials, the theory predicts that any elastic particle is stably trapped, although increasing 
the acoustic power may be necessary to overcome the gravitational force at this scale. The trapping beam seems robust to other shapes, as demonstrated experimentally for a system of two spheres. Regarding the particle size, beads up to a third of the wavelength in diameter were successively trapped, but larger ones are predicted to be expelled. Small beads are also trappable. However, the gradient force decreases faster for small particles than the drag initiated by acoustic streams. The best solution is to scale up the frequency to keep a radius-to-wavelength ratio sufficiently large. A driving frequency approaching $f=100 \mathrm{MHz}$ [54] would give access to beads in the microscopic range.

It seems particularly relevant and appealing to extend the "microscopic handle" technique to acoustical tweezers to apply and precisely measure forces involved at the cellular scale in biophysical processes such as morphogenesis, organogenesis and cell adhesion $[55,56]$.

*jean-louis.thomas@upmc.fr

[1] A. Ashkin, Acceleration and Trapping of Particles by Radiation Pressure, Phys. Rev. Lett. 24, 156 (1970).

[2] A. Ashkin, How it all began, Nat. Photonics 5, 316 (2011).

[3] A. Ashkin, J. M. Dziedzic, J. E. Bjorkholm, and S. Chu, Observation of a single-beam gradient force optical trap for dielectric particles, Opt. Lett. 11, 288 (1986).

[4] A. Ashkin, J. M. Dziedzic, and T. Yamane, Optical trapping and manipulation of single cells using infrared laser beams, Nature (London) 330, 769 (1987).

[5] D. Grier, A revolution in optical manipulation, Nature (London) 424, 810 (2003).

[6] K. Dholakia and T. Čižmár, Shaping the future of manipulation, Nat. Photonics 5, 335 (2011).

[7] M. Padgett and R. Bowman, Tweezers with a twist, Nat. Photonics 5, 343 (2011).

[8] A. Aspect, E. Arimondo, R. Kaiser, N. Vansteenkiste, and C. Cohen-Tannoudji, Laser Cooling below the One-Photon Recoil Energy by Velocity-Selective Coherent Population Trapping, Phys. Rev. Lett. 61, 826 (1988).

[9] K. Svoboda and S. M. Block, Biological applications of optical forces, Annu. Rev. Biophys. Biomol. Struct. 23, 247 (1994).

[10] K. C. Neuman and A. Nagy, Single-molecule force spectroscopy: Optical tweezers, magnetic tweezers and atomic force microscopy, Nat. Methods 5, 491 (2008).

[11] A. Ashkin, Applications of laser radiation pressure, Science 210, 1081 (1980).

[12] A. Eller, Force on a bubble in a standing acoustic wave, J. Acoust. Soc. Am. 43, 170 (1968).

[13] E. H. Trinh, Compact acoustic levitation device for studies in fluid dynamics and material science in the laboratory and microgravity, Rev. Sci. Instrum. 56, 2059 (1985).

[14] R. Apfel, Acoustic levitation for studying liquids and biological materials, J. Acoust. Soc. Am. 70, 636 (1981).

[15] D. Foresti and D. Poulikakos, Acoustophoretic Contactless Elevation, Orbital Transport and Spinning of Matter in Air, Phys. Rev. Lett. 112, 024301 (2014).
[16] T. Laurell, F. Petersson, and A. Nilsson, Chip integrated strategies for acoustic separation and manipulation of cells and particles, Chem. Soc. Rev. 36, 492 (2007).

[17] P. B. Muller, M. Rossi, Á. G. Marín, R. Barnkob, P. Augustsson, T. Laurell, C. J. Kähler, and H. Bruus, Ultrasound-induced acoustophoretic motion of microparticles in three dimensions, Phys. Rev. E 88, 023006 (2013).

[18] X. Ding, S.-C. S. Lin, B. Kiraly, H. Yue, S. Li, I.-K. Chiang, J. Shi, S. J. Benkovic, and T. J. Huang, On-chip manipulation of single microparticles, cells, and organisms using surface acoustic waves, Proc. Natl. Acad. Sci. U.S.A. 109, 11105 (2012).

[19] S. B. Q. Tran, P. Marmottant, and P. Thibault, Fast acoustic tweezers for the two-dimensional manipulation of individual particles in microfluidic channels, Appl. Phys. Lett. 101, 114103 (2012).

[20] C. R. P. Courtney, B. W. Drinkwater, C. E. M. Demore, S. Cochran, A. Grinenko, and P.D. Wilcox, Dexterous manipulation of microparticles using Bessel-function acoustic pressure fields, Appl. Phys. Lett. 102, 123508 (2013).

[21] F. Guo, P. Li, J. B. French, Z. Mao, H. Zhao, S. Li, N. Nama, J. R. Fick, S. J. Benkovic, and T. J. Huang, Controlling cell-cell interactions using surface acoustic waves, Proc. Natl. Acad. Sci. U.S.A. 112, 43 (2015).

[22] C. R. P. Courtney, C. E. M. Demore, H. Wu, A. Grinenko, P. D. Wilcox, S. Cochran, and B. W. Drinkwater, Independent trapping and manipulation of microparticles using dexterous acoustic tweezers, Appl. Phys. Lett. 104, 154103 (2014).

[23] J. Wu, Acoustical tweezers, J. Acoust. Soc. Am. 89, 2140 (1991).

[24] J. Lee et al., Single beam acoustic trapping, Appl. Phys. Lett. 95, 073701 (2009).

[25] Y. Li, C. Lee, R. Chen, Q. Zhou, and K. K. Shung, A feasibility study of in vivo applications of single beam acoustic tweezers, Appl. Phys. Lett. 105, 173701 (2014).

[26] D. Baresch, J.-L. Thomas, and R. Marchiano, Spherical vortex beams of high radial degree for enhanced singlebeam tweezers, J. Appl. Phys. 113, 184901 (2013).

[27] P. L. Marston, Axial radiation force of a Bessel beam on a sphere and direction reversal of the force, J. Acoust. Soc. Am. 120, 3518 (2006).

[28] P. L. Marston, Radiation force of a helicoidal Bessel beam on a sphere, J. Acoust. Soc. Am. 125, 3539 (2009).

[29] D. Baresch, J.-L. Thomas, and R. Marchiano, Threedimensional acoustic radiation force on an arbitrarily located elastic sphere, J. Acoust. Soc. Am. 133, 25 (2013).

[30] B. Maheu, G. Gouesbet, and G. Grehan, A concise presentation of the generalized Lorenz-Mie theory for arbitrary location of the scatterer in an arbitrary incident profile, J. Opt. 19, 59 (1988).

[31] See Supplemental Material at http://link.aps.org/ supplemental/10.1103/PhysRevLett.116.024301 for further details on experiments, calculations and movies, which includes Refs. [32-38].

[32] O. Sapozhnikov and M. Bailey, Radiation force of an arbitrary acoustic beam on an elastic sphere in a fluid, J. Acoust. Soc. Am. 133, 661 (2013). 
[33] O. Farsund and B. Felderhof, Force, torque, and absorbed energy for a body of arbitrary shape and constitution in an electromagnetic radiation field, Physica (Amsterdam) 227A, 108 (1996).

[34] L. P. Gor'kov, On the forces acting on a small particle in an acoustical field in an ideal fluid, Sov. Phys.-Doklady 6, 773 (1962).

[35] M. Tanter, J.-F. Aubry, J. Gerber, J.-L. Thomas, and M. Fink, Optimal focusing by spatio-temporal inverse filter. I. Basic principles, J. Acoust. Soc. Am. 110, 37 (2001).

[36] I. S. Gradshteyn and I. M. Ryzhik, Table of Integrals, Series, and Products (Academic Press, Elsevier, 2007), Chap. 8.

[37] J. J. Faran, Sound scattering by solid cylinders and spheres, J. Acoust. Soc. Am. 23, 405 (1951).

[38] C. T. Crowe, J. D. Schwarzkopf, M. Sommerfeld, and Y. Tsuji, Multiphase Flows with Droplets and Particles, 2nd ed. (CRC Press, Taylor \& Francis Group, Boca Raton, 2011), Chap. 4.

[39] B. T. Hefner and P. L. Marston, An acoustical helicoidal wave transducer with applications for the alignment of ultrasonic and underwater systems, J. Acoust. Soc. Am. 106, 3313 (1999).

[40] J.-L. Thomas and R. Marchiano, Pseudo Angular Momentum and Topological Charge Conservation for Nonlinear Acoustical Vortices, Phys. Rev. Lett. 91, 244302 (2003).

[41] R. Marchiano and J.-L. Thomas, Doing Arithmetic With Nonlinear Acoustic Vortices, Phys. Rev. Lett. 101, 064301 (2008).

[42] K. Volke-Sepúlveda, A. Santillán, and R. Bullosa, Transfer of Angular Momentum to Matter from Acoustical Vortices in Free Space, Phys. Rev. Lett. 100, 024302 (2008).

[43] K. Skeldon, C. Wilson, M. Edgar, and M. Padgett, An acoustic spanner and its associated rotational Doppler shift, New J. Phys. 10, 013018 (2008).

[44] C. E. M. Demore, Z. Yang, A. Volovick, S. Cochran, M. P. MacDonald, and G. C. Spalding, Mechanical Evidence of the Orbital Angular Momentum to Energy Ratio of Vortex Beams, Phys. Rev. Lett. 108, 194301 (2012).

[45] A. Anhäuser, R. Wunenburger, and E. Brasslet, Acoustic Rotational Manipulation Using Orbital Angular Momentum Transfer, Phys. Rev. Lett. 109, 034301 (2012).

[46] Z. Hong, J. Zhang, and B. Drinkwater, Observation of Orbital Angular Momentum Transfer from Bessel-Shaped Acoustic Vortices to Diphasic Liquid-Microparticle Mixtures, Phys. Rev. Lett. 114, 214301 (2015).

[47] C. Eckart, Vortices and streams caused by sound waves, Phys. Rev. 73, 68 (1948).

[48] A. Riaud, M. Baudoin, J.-L. Thomas, and O. B. Matar, Cyclones and attractive streaming generated by acoustical vortices, Phys. Rev. E 90, 013008 (2014).

[49] G. T. Silva and H. Bruus, Acoustic interaction forces between small particles in an ideal fluid, Phys. Rev. E 90, 063007 (2014).

[50] E. R. Dufresne and D. G. Grier, Optical tweezer arrays and optical substrates created with diffractive optics, Rev. Sci. Instrum. 69, 1974 (1998).

[51] D. B. Ruffner and D. G. Grier, Optical Conveyors: A Class of Active Tractor Beams, Phys. Rev. Lett. 109, 163903 (2012).

[52] L. Zhang and P. L. Marston, Acoustic radiation torque on small objects in viscous fluids and connection with viscous dissipation, J. Acoust. Soc. Am. 136, 2917 (2014).

[53] L. Zhang and P. L. Marston, Angular momentum flux of nonparaxial acoustic vortex beams and torques on axisymmetric objects, Phys. Rev. E 84, 065601 (2011).

[54] K. H. Lam, H.-S. Hsu, Y. Li, C. Lee, A. Lin, Q. Zhou, E.S. Kim, and K. K. Shung, Ultrahigh frequency lensless ultrasonic transducers for acoustic tweezers application, Biotechnol. Bioeng. 110, 881 (2013).

[55] N. Desprat, W. Supatto, P.-A. Pouille, E. Beaurepaire, and E. Farge, Tissue deformation modulates twist expression to determine anterior midgut differentiation in Drosophila Embryos, Dev. Cell 15, 470 (2008).

[56] T. Brunet et al., Evolutionary conservation of early mesoderm specification by mechanotransduction in Bilateria, Nat. Commun. 4, 2821 (2013). 